\title{
Scale-dependent correlation of seabirds with schooling fish in a coastal ecosystem
}

\author{
David C. Schneider \& John F. Piatt \\ Newfoundland Institute for Cold Ocean Science, Memorial University of Newfoundland, St. John's, \\ Newioundland A1B 3X7, Canada
}

\begin{abstract}
The distribution of piscivorous seabirds relative to schooling fish was investigated by repeated censusing of 2 intersecting transects in the Avalon Channel, which carries the Labrador Current southward along the east coast of Newfoundland. Murres (primarily common murres Uria aalge), Atlantic puffins Fratercula arctica, and schooling fish (primarily capelin Mallotus villosus) were highly aggregated at spatial scales ranging from 0.25 to $15 \mathrm{~km}$. Patchiness of murres, puffins and schooling fish was scale-dependent, as indicated by significantly higher variance-to-mean ratios at large measurement distances than at the minimum distance, $0.25 \mathrm{~km}$. Patch scale of puffins ranged from 2.5 to $15 \mathrm{~km}$, of murres from 3 to $8.75 \mathrm{~km}$, and of schooling fish from 1.25 to $15 \mathrm{~km}$. Patch scale of birds and schooling fish was similar in 6 out of 9 comparisons. Correlation between seabirds and schooling birds was significant at the minimum measurement distance in 6 out of 12 comparisons. Correlation was scale-dependent, as indicated by significantly higher coefficients at large measurement distances than at the minimum distance. Tracking scale, as indicated by the maximum significant correlation between birds and schooling fish, ranged from 2 to $6 \mathrm{~km}$. Our analysis showed that extended aggregations of seabirds are associated with extended aggregations of schooling fish and that correlation of these marine carnivores with their prey is scale-dependent.
\end{abstract}

\section{INTRODUCTION}

Marine birds form aggregations that range from a few metres to tens of kilometres in lateral extent. Aggregations ranging from 5 to $50 \mathrm{~km}$ in chord length have been reported in the Bering Sea (Schneider 1982, Kinder et al. 1983, Woodby 1984), the California Current (Briggs et al. 1984), and the Benguela Current (Schneider \& Duffy 1985). The existence of these aggregations suggests that seabirds may be correlated with prey at a similar scale, but this has not been tested directly.

We investigated the distribution of murres (primarily the common murre Uria aalgel and Atlantic puffins Fratercula arctica relative to schooling fish at Witless Bay, the site of a major seabird colony in the northwest Atlantic. This colony consists of 3 closely spaced islands on the western side of the Avalon Channel, a relatively deep $(>150 \mathrm{~m})$ nearshore trough that funnels the inshore arm of the Labrador Current southward along the east coast of Newfoundland. Capelin spawn along this coast from late June into mid-July (Templeman 1948), and during this period they are the most abundant schooling fish nearshore. Capelin are the most important prey in the diets of murres and puffins at Witless Bay (Mahoney 1979, Brown \& Nettleship 1984, J. Piatt unpubl data).

We investigated variability in the abundance of birds and schooling fish by making repeated surveys of 2 transects at Witless Bay during the summer of 1984 . Our objectives were to determine (1) whether variability in the abundance of birds and schooling fish was scale dependent; (2) whether birds and fish were locally correlated; and (3) whether correlation was scaledependent.

\section{METHODS}

Surveys were made along an offshore transect running eastward from Green Island into the Avalon Channel and along a coastal transect running parallel to the coastline from Bay Bulls to Cape Broyle (Fig. 1). The offshore transect was surveyed 4 times and the coastal transect was surveyed 3 times during the summer of 1984

Bird counts were made from a $12 \mathrm{~m}$ fishing vessel 


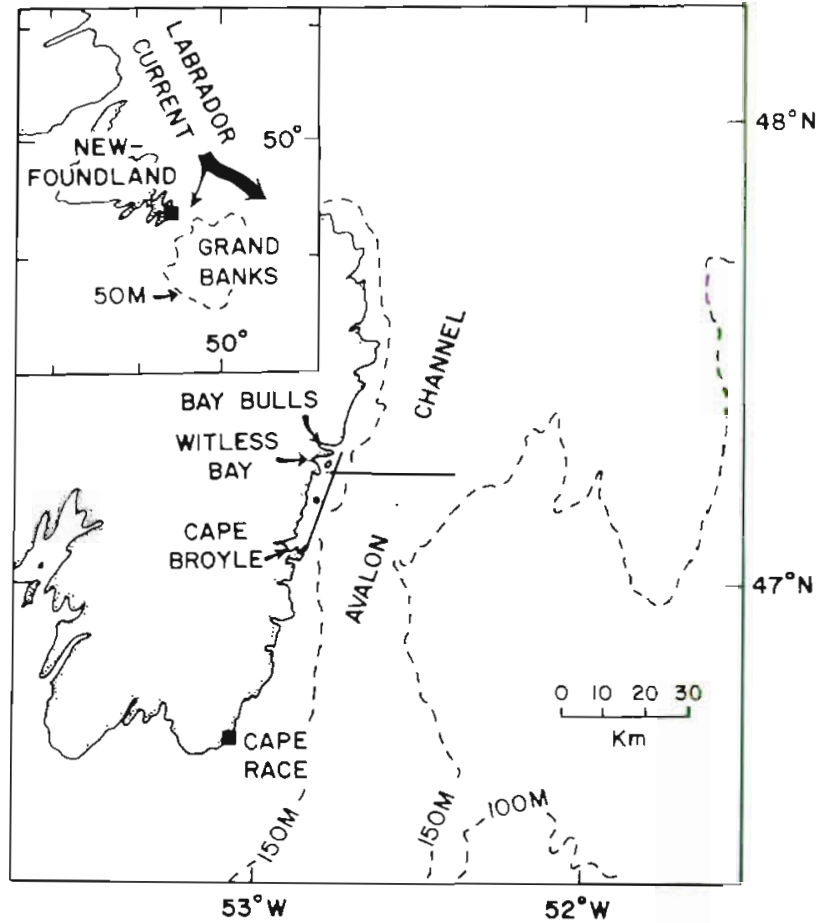

Fig. 1. Location of transects in Northwest Atlantic

equipped with a depth sounder for acoustic recording of schooling fish. The depth sounder (Kelvin-Hughes Mark 2, $42 \mathrm{kHz}$ ) was mounted on the bottom of the vessel, approximately $1 \mathrm{~m}$ below the water line. Cone angle was $33^{\circ}$ and effective recording depth ranged from $2 \mathrm{~m}$ below the surface down to the ocean floor. Murres and puffins on the water were counted in a semicircular zone from the vessel forward. The area of the observation zone was 0.79 ha, with a radius of $50 \mathrm{~m}$. Counts were recorded to the nearest second and grouped into 1 min intervals for comparison with echograms from the depth sounder.

The echogram from each acoustic survey was divided horizontally into 1 min intervals and vertically into $10 \mathrm{~m}$ depth intervals. The most important criteria were school shape and definition at the boundary. Schooling fish were identified according to the criteria of Forbes \& Nakken (1972). The registration intensity of schooling fish within each $1 \mathrm{~min}(250 \mathrm{~m})$ by $10 \mathrm{~m}$ block was scored visually on a scale of 0 to 9 . This score was squared to make it proportional to fish abundance (Forbes \& Nakken 1972), and then summed by depth categories to obtain an index of relative abundance during each minute of survey. The dynamic range of the echograms was low and most of the registrations were black or nearly so. The squaring of registration scores had little effect on the index of relative abundance, which was primarily a function of school size rather than density.
Spatial variability in the abundance of murres, puffins, and schooling fish was measured by computing a variance-to-mean ratio, $I^{\prime}$, for measurement distances ranging from 0.25 to $T / 2 \mathrm{~km}$, where $T$ was the total number of kilometres per transect.

$$
I^{\prime}(F)=s^{2}(F) \bar{x}^{-1}(F)
$$

where $\mathrm{F}=$ measurement distance $\left(\mathrm{km} \mathrm{cycle}^{-1}\right)$;

$\overline{\mathrm{x}}(\mathrm{F})=\mathrm{n}^{-1} \sum^{\mathrm{n}} \sum^{\mathrm{F}} \mathrm{x}_{i i}$

$\mathrm{s}^{2}(\mathrm{~F})=(\mathrm{n}-1)^{-1}\left(\sum^{\mathrm{n}}\left[\sum^{\mathrm{F}} \mathrm{x}_{\mathrm{i}}\right]^{2}-\mathrm{n} \overline{\mathrm{x}}(\mathrm{F})^{2}\right) ;$

$\mathrm{x}_{1}=$ bird count or fish score at the minimum measurement distance, $0.25 \mathrm{~km}$;

$\mathrm{n}=$ sampling frequency (cycles transect ${ }^{-1}$ ).

Computations were restricted to values of $\mathrm{F}$ corresponding to integral values of $\mathrm{n}$ within each survey. Strength of association of birds with schooling fish was measured by computing the Pearson product moment correlation coefficient, $r$, as a function of measurement distance. Correlations were computed for the same measurement distances as $\mathrm{I}^{\prime}(\mathrm{F})$.

The seabird data could not be described by standard statistical models such as the normal and negative binomial distributions, so randomization tests were used to evaluate statistical significance. The first null hypothesis, scale-independent variability, $\mathrm{H}_{0}: \mathrm{I}^{\prime}(0.25)$ $=I^{\prime}(F)$, was rejected in favor of the alternative hypothesis, $\mathrm{H}_{\mathrm{A}}: \mathrm{I}^{\prime}(0.25)<\mathrm{I}^{\prime}(\mathrm{F})$, if an observed value of $I^{\prime}(F)$ exceeded 95 out of 100 values of $I^{\prime}(F)$ obtained by randomizing the 1 min counts, $x_{i}$. A Fortran subroutine (GGPER) from the International Mathematics and Statistics Library (IMSL 1982) was used to obtain random permutations of the location index (i), and these were used to reassign each $1 \mathrm{~min}(0.25 \mathrm{~km})$ count to a new location along the transect. Patch scale was defined as the measurement distance corresponding to the maximum value of $I^{\prime}(F)$ that was significantly greater than I' 10.25$)$.

Correlation of puffins and murres with fish at the smallest measurement distance was tested for significance by comparing the observed value of $\mathrm{s}(0.25)$ to 100 values obtained by randomizing $1 \mathrm{~min}(0.25 \mathrm{~km})$ bird counts with respect to location. Fish abundance was not randomized in this analysis. The second null hypothesis, $\mathrm{H}_{0}: \mathrm{r}(0.25)=0$, was rejected if an observed value of $r(0.25)$ was greater than 95 out of 100 values of $r(0.25)$ from randomized data.

The third null hypothesis, scale-independent correlation, $H_{0}: r(0.25)=r(F)$, was rejected in favor of the alternative hypothesis, $H_{A}: r(0.25)<r(F)$ if an observed value of $r(F)$ was greater than 95 out of 100 values obtained from randomized data. Tracking scale was defined as the measurement distance correspond- 
Table 1. Abundance of murres, puffins, and schooling fish along offshore and coastal transects in the Avalon Channel in 1984. Bird counts are number seen on the water within $50 \mathrm{~m}$ of either side of the vessel. Relative abundance of fish based on visually graded echograms. Ship speed $=250 \mathrm{~m} \mathrm{~min}^{-1}$ Censusing rate $=2.5 \mathrm{ha} \mathrm{min}^{-1}$. Local time (NST) $=$ Universal time $-2.5 \mathrm{~h}$

\begin{tabular}{|c|c|c|c|c|c|}
\hline Date & Start (NST) & Length $(\mathrm{km})$ & Murres & Puffins & Schooling fish \\
\hline \multicolumn{6}{|c|}{ Offshore transect } \\
\hline $30 \mathrm{May}$ & 0834 & 30 & 249 & 145 & 9 \\
\hline $6 \mathrm{Jun}$ & 0835 & 25 & 12 & 1 & 99 \\
\hline $16 \mathrm{Jul}$ & 0753 & 30 & 41 & 8 & 38 \\
\hline 14 Aug & 0735 & 30 & 0 & 52 & 13 \\
\hline \multicolumn{6}{|c|}{ Coastal transect } \\
\hline 14 Jun & 0707 & 15 & 48 & 121 & 35 \\
\hline $8 \mathrm{Jul}$ & 1016 & 30 & 746 & 233 & 286 \\
\hline $18 \mathrm{Jul}$ & 0750 & 17.5 & 226 & 265 & 159 \\
\hline
\end{tabular}

ing to the maximum value of $\mathrm{r}(\mathrm{F})$ that was significantly greater than $\mathrm{r}(0.25)$.

Type I eror, erroneous rejection of a null hypothesis, was estimated by Monte Carlo methods (Schreider 1966). Values of $I^{\prime}(F)$ and $r(F)$ were obtained from 100 randomizations of a single set of data, and these were tested for significance. The expected number of Type I errors was 5 in 100 trials.

\section{RESULTS}

\section{Abundance of murres, puffins, and schooling fish}

The number of murres and puffins recorded along the 2 transects varied from survey to survey (Table 1). The highest fish abundances were recorded along the coastal transect during the spawning season in July (Table 1). The highest murre and puffin abundances were also recorded along the coastal transect in July.

Distribution of murres, puffins, and fish along the offshore transect (Table 2) was a function of distance from land. Murres and puffins were more abundant nearshore than offshore during all 4 offshore surveys. Fish were more abundant nearshore than offshore during 3 of the 4 surveys (Table 3 ).

\section{Type I error for randomization tests}

Fig. 2 compares the observed values of $I^{\prime}(F)$ to values of $I^{\prime}(F)$ obtained from 5 randomizations of the data from the 30 May survey. We expected values of $I^{\prime}$ (F) from randomized data to be scale independent, i.e. $I^{\prime}(F)=I^{\prime}(0.25)$. Inspection of Fig. 2 shows that randomized values of $I^{\prime}(F)$ fluctuated around the expected value, $I^{\prime}(0.25)$, while observed values of $I^{\prime}(F)$ tended to exceed the expected value. Randomized values of I' $(F)$ in puffins did appear to be biased toward low values at large measurement distances, possibly because of bias introduced by small sample sizes (Reed 1983). None of the low values of $\mathrm{I}^{\prime}(\mathrm{F})$ in the randomized puffin data were significant, which indicated that Monte Carlo tests were not sensitive to bias at large measurement distances. The expected number of Type I errors in the 5 randomizations in Fig. 3 was 10.5 (5\% of 210 tests); the observed number was 6.

Type I error rate for each of the 3 null hypotheses was estimated from 100 randomizations of the 30 May data. Type I error rate for randomization tests was close to the expected rate of $5 \%$ (Table 4 ). Type I error for randomization tests of the first null hypothesis, scaleindependent variability, averaged $4.1 \%$ in murres, $5.6 \%$ in puffins, and $3.4 \%$ in fish. Type I error for the

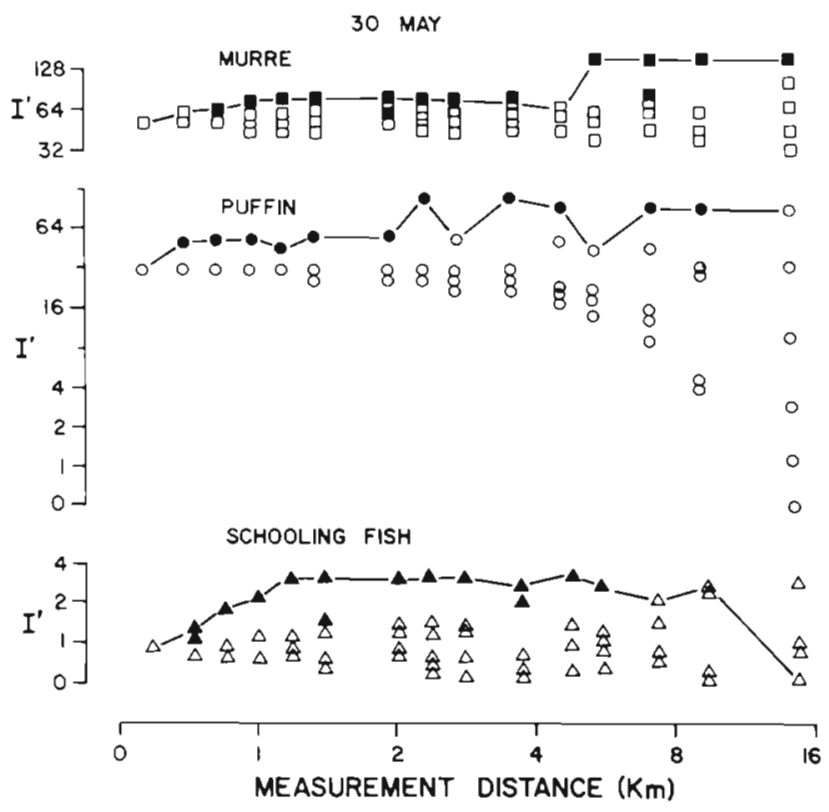

Fig. 2. Variance-to-mean ratios, I', for observed data (connected by lines) and randomized data (not connected by lines) on murres, puffins, and schooling fish during first survey of offshore transect, on 30 May. Statistical significance indicated by solid symbols $(p<0.05)$ and open symbols $(p>0.05)$ 
Table 2. Distribution of murres (M), schooling fish $(F)$, and puffins (P) along 4 offshore transects in 1984

\begin{tabular}{|c|c|c|c|c|c|c|c|c|c|c|c|c|c|c|c|c|c|c|c|c|c|c|c|c|c|c|c|}
\hline \multirow{2}{*}{$\begin{array}{l}\text { Time } \\
\text { (min) }\end{array}$} & \multicolumn{3}{|c|}{30 May } & \multicolumn{3}{|c|}{6 Jun } & \multicolumn{3}{|c|}{$16 \mathrm{Jul}$} & 14 & Aug & & Distance & Time & 30 & $M$ & & & $\mathrm{Ju}$ & & & $\mathrm{J}$ & & & $A l$ & & Distance \\
\hline & M & $\mathrm{F}$ & $\mathrm{P}$ & M & & & M & & & & & & $(\mathrm{km})$ & $(\min )$ & $M$ & $F$ & $\mathrm{P}$ & $M$ & $F$ & $\mathrm{P}$ & $M$ & $\mathrm{~F}$ & P & $M$ & $\mathrm{~F}$ & $\mathrm{P}$ & \\
\hline 0 & 0 & 1 & 0 & 0 & 0 & 0 & 0 & 2 & 0 & 0 & 00 & & 0 & & 0 & 0 & 0 & 0 & 1 & 0 & 0 & 0 & 0 & 0 & 0 & 0 & \\
\hline & 11 & 1 & 0 & 0 & 0 & 0 & 0 & 1 & 2 & 0 & 00 & & & & 2 & 0 & 0 & 0 & 1 & 0 & 0 & 0 & 0 & 0 & 0 & 0 & \\
\hline & 26 & 1 & 0 & 0 & 0 & 0 & 0 & 2 & 0 & 0 & $\begin{array}{ll}0 & 0\end{array}$ & & & & 0 & 0 & 0 & 0 & 1 & 0 & 0 & 0 & 0 & 0 & 0 & 0 & \\
\hline & 10 & 1 & 0 & 0 & 0 & 0 & 0 & 2 & 0 & 0 & 12 & & & & 0 & 0 & 0 & 0 & 1 & 0 & 0 & 0 & 0 & 0 & 0 & 0 & \\
\hline & 3 & 1 & 2 & 0 & 1 & 0 & 0 & 2 & 1 & 0 & 21 & & & & 0 & 0 & 0 & 0 & 1 & 0 & 0 & 0 & 0 & 0 & 0 & 0 & \\
\hline & 6 & 0 & 0 & 0 & 1 & 0 & 0 & 0 & 0 & 0 & 05 & & & & 0 & 0 & 0 & 0 & 1 & 0 & 0 & 0 & 0 & 0 & 0 & 0 & \\
\hline & 5 & 0 & 0 & 0 & 1 & 0 & 0 & 0 & 0 & 0 & 13 & & & & 0 & 0 & 0 & 0 & 1 & 0 & 0 & 0 & 0 & 0 & 0 & 1 & \\
\hline & 4 & 0 & 0 & 0 & 1 & 0 & 0 & 0 & 0 & 0 & $\begin{array}{ll}0 & 0\end{array}$ & & & & 1 & 0 & 0 & 0 & 1 & 0 & 1 & 0 & 0 & 0 & 0 & 0 & \\
\hline & 0 & 0 & 0 & 0 & 1 & 0 & 0 & 0 & 0 & 0 & 02 & & & & 0 & 0 & 0 & 0 & $\hat{1}$ & 0 & 0 & 0 & 0 & 0 & 0 & 0 & \\
\hline & 0 & 0 & 0 & 0 & 1 & 0 & 7 & 3 & 0 & 0 & 23 & & & & 0 & 0 & 0 & 0 & 1 & 0 & 0 & 1 & 0 & 0 & 0 & 0 & \\
\hline & 4 & 0 & 0 & 0 & 1 & 0 & 0 & 1 & 0 & 0 & 12 & & & & 0 & 0 & 2 & 0 & 1 & 0 & 2 & 0 & 0 & 0 & 0 & 1 & \\
\hline & 1 & 0 & 0 & 0 & 1 & 0 & 0 & 3 & 0 & 0 & 05 & & & & 0 & 0 & 0 & 0 & 1 & 0 & 1 & 0 & 0 & 0 & 0 & 0 & \\
\hline & 0 & 0 & 0 & 0 & 1 & 0 & 0 & 2 & 0 & 0 & $\begin{array}{ll}0 & 1\end{array}$ & & & & 1 & 0 & 0 & 0 & 1 & 0 & 0 & 0 & 0 & 0 & 0 & 1 & \\
\hline & 0 & 0 & 0 & 1 & 1 & 0 & 3 & 2 & 0 & 0 & 00 & & & & 0 & 0 & 0 & 0 & 1 & 0 & 0 & 0 & 0 & 0 & 0 & 0 & \\
\hline & 0 & 0 & 0 & 0 & 1 & 0 & 0 & 1 & 0 & 0 & $\begin{array}{ll}0 & 1\end{array}$ & & & & 0 & 0 & 0 & 0 & 1 & 0 & 0 & 0 & 0 & 0 & 0 & 0 & \\
\hline & 0 & 0 & 0 & 1 & 1 & 0 & 1 & 1 & 0 & 0 & $\begin{array}{lll}0 & 0\end{array}$ & & & & 0 & 0 & 0 & 0 & 1 & 0 & 3 & 0 & 0 & 0 & 0 & 0 & \\
\hline & 1 & 0 & 0 & 0 & 4 & 0 & 1 & 1 & 0 & 0 & 02 & & & & 1 & 0 & 0 & 0 & 1 & 0 & 1 & 0 & 0 & 0 & 0 & 0 & \\
\hline & 0 & 0 & 0 & 0 & 1 & 0 & 2 & 1 & 2 & 0 & $\begin{array}{ll}0 & 1\end{array}$ & & & & 7 & 0 & 0 & 0 & 1 & 0 & 1 & 0 & 1 & 0 & 0 & 0 & \\
\hline & 0 & 0 & 0 & 0 & 1 & 0 & 0 & 1 & 0 & 0 & 02 & & & & 5 & 0 & 2 & 0 & 1 & 0 & 3 & 0 & 0 & 0 & 0 & 0 & \\
\hline 20 & 5 & 0 & 0 & 0 & 1 & 0 & 0 & 1 & 0 & 0 & 00 & & 5 & 80 & 0 & 0 & 0 & 0 & 1 & 0 & 1 & 0 & 0 & 0 & 0 & 0 & 20 \\
\hline & 2 & 0 & 2 & 0 & 1 & 0 & 0 & 1 & 0 & 0 & $\begin{array}{ll}0 & 1\end{array}$ & & & & 0 & 0 & 0 & 0 & 1 & 0 & 0 & 0 & 0 & 0 & 0 & 0 & \\
\hline & 9 & 0 & 0 & 1 & 1 & 0 & 1 & 1 & 0 & 0 & $\begin{array}{lll}0 & 0\end{array}$ & ) & & & 0 & 0 & 0 & 0 & 1 & 0 & 0 & 0 & 1 & 0 & 0 & 1 & \\
\hline & 114 & 0 & 0 & 0 & 1 & 0 & 0 & 1 & 0 & 0 & 21 & L & & & 0 & 0 & 0 & 0 & 1 & 0 & 0 & 0 & 0 & 0 & 0 & 0 & \\
\hline & 6 & 0 & 36 & 0 & 1 & 0 & 0 & 1 & 0 & 0 & $\begin{array}{lll}0 & 0\end{array}$ & 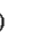 & & & 3 & 1 & 0 & 0 & 1 & 0 & 2 & 0 & 0 & 0 & 0 & 0 & \\
\hline & 1 & 0 & 26 & 0 & 1 & 0 & 2 & 0 & 0 & 0 & $\begin{array}{ll}0 & 1\end{array}$ & & & & 0 & 0 & 0 & 0 & 1 & 0 & 0 & 0 & 0 & 0 & 0 & 0 & \\
\hline & 0 & 0 & 55 & 0 & 1 & 0 & 1 & 1 & 0 & 0 & 00 & & & & 2 & 0 & 1 & 2 & 1 & 0 & 0 & 0 & 0 & 0 & 0 & 0 & \\
\hline & 0 & 0 & 6 & 0 & 1 & 0 & 0 & 0 & 0 & 0 & $\begin{array}{ll}0 & 1\end{array}$ & & & & 0 & 0 & 0 & 0 & 1 & 0 & 0 & 0 & 0 & 0 & 0 & 1 & \\
\hline & 1 & 0 & 0 & 0 & 1 & 0 & 1 & 0 & 0 & 0 & 00 & & & & 0 & 0 & 1 & 0 & 1 & 0 & 0 & 2 & 0 & 0 & 0 & 0 & \\
\hline & 0 & 0 & 1 & 0 & 1 & 0 & 0 & 0 & 0 & 0 & 02 & & & & 0 & 0 & 0 & 0 & 1 & 0 & 0 & 0 & 0 & 0 & 0 & 0 & \\
\hline & 0 & 0 & 0 & 0 & 1 & 0 & 0 & 0 & 0 & 0 & 00 & & & & 0 & 0 & 0 & 0 & 1 & 0 & 0 & 0 & 0 & 0 & 0 & 1 & \\
\hline & 0 & 0 & 0 & 0 & 1 & 0 & 0 & 0 & 0 & 0 & 00 & & & & 0 & 0 & 0 & 0 & 1 & 0 & 0 & 0 & 0 & 0 & 0 & 0 & \\
\hline & 0 & 0 & 0 & 0 & $\hat{1}$ & 0 & 0 & 0 & 0 & 0 & 00 & ) & & & 0 & 0 & 0 & 0 & 1 & 0 & 0 & 0 & 0 & 0 & 0 & 0 & \\
\hline & 0 & 0 & 0 & 0 & 1 & 0 & 0 & 0 & 0 & 0 & 00 & 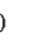 & & & 0 & 0 & 0 & 0 & 1 & 0 & 1 & 0 & 0 & 0 & 0 & 0 & \\
\hline & 0 & 0 & 0 & 0 & 1 & 0 & 0 & 0 & 0 & 0 & $\begin{array}{ll}0 & 1\end{array}$ & & & & 1 & 0 & 0 & 0 & 1 & 0 & 0 & 0 & 0 & 0 & 0 & 0 & \\
\hline & 0 & 0 & 0 & 1 & 1 & 0 & 0 & 0 & 0 & 0 & 00 & & & & 0 & 0 & 0 & 0 & 1 & 0 & 0 & 0 & 0 & 0 & 0 & 0 & \\
\hline & 0 & 0 & 0 & 0 & 1 & 0 & 0 & 0 & 0 & 0 & 00 & 0 & & & 0 & 0 & 0 & 0 & 1 & 1 & 0 & 0 & 0 & 0 & 0 & 0 & \\
\hline & 0 & 0 & 0 & 0 & 1 & 0 & 0 & 1 & 0 & 0 & $\begin{array}{ll}0 & 1\end{array}$ & & & & 0 & 0 & 0 & 0 & 1 & 0 & 0 & 0 & 0 & 0 & 0 & 0 & \\
\hline & 0 & 0 & 0 & 0 & 1 & 0 & 0 & 0 & 0 & 0 & 00 & & & & 0 & 0 & 0 & 0 & 1 & 0 & 0 & 0 & 0 & 0 & 0 & 0 & \\
\hline & 0 & 0 & 0 & 0 & 1 & 0 & 0 & 0 & 0 & 0 & $\begin{array}{ll}0 & 1\end{array}$ & & & & 0 & 0 & 0 & 0 & 1 & 0 & 0 & 0 & 0 & 0 & 0 & 1 & \\
\hline 40 & 0 & 0 & 0 & 0 & 1 & 0 & 1 & 0 & 0 & 0 & 00 & & 10 & 100 & 0 & 0 & 0 & 0 & 1 & 0 & 0 & 0 & 0 & 0 & 0 & 0 & 25 \\
\hline & 0 & 0 & 0 & 0 & 1 & 0 & 0 & 0 & 0 & 0 & 00 & 9 & & & 0 & 0 & 0 & & & & 0 & 0 & 0 & 0 & 0 & 0 & \\
\hline & 0 & 0 & 0 & 0 & 1 & 0 & 0 & 0 & 0 & 0 & 00 & 0 & & & 0 & 0 & 0 & & & & 0 & 0 & 0 & 0 & 0 & 0 & \\
\hline & 0 & 0 & 0 & 0 & 1 & 0 & 0 & 0 & 0 & 0 & 02 & & & & 0 & 0 & 0 & & & & 0 & 0 & 0 & 0 & 0 & 0 & \\
\hline & 0 & 0 & 0 & 0 & 1 & 0 & 0 & 0 & 0 & 0 & 00 & & & & 0 & 1 & 0 & & & & 0 & 0 & 0 & 0 & 0 & 0 & \\
\hline & 4 & 0 & 0 & 0 & 1 & 0 & 0 & 0 & 0 & 0 & $\begin{array}{ll}0 & 0\end{array}$ & & & & 2 & 0 & 3 & & & & 0 & 0 & 0 & 0 & 0 & 0 & \\
\hline & 0 & 0 & 0 & 0 & 1 & 0 & 0 & 0 & 0 & 0 & 00 & 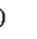 & & & 0 & 0 & 4 & & & & 0 & 0 & 0 & 0 & 0 & 0 & \\
\hline & 1 & 0 & 0 & 0 & 1 & 0 & 0 & 0 & 0 & 0 & 00 & 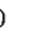 & & & 0 & 0 & 0 & & & & 0 & 0 & 0 & 0 & 0 & 0 & \\
\hline & 0 & 0 & 0 & 1 & 1 & 0 & 0 & 0 & 0 & 0 & 00 & 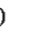 & & & 0 & 0 & 0 & & & & 0 & 0 & 0 & 0 & 0 & 1 & \\
\hline & 1 & 0 & 0 & 0 & 1 & 0 & 0 & 0 & 0 & 0 & $0 \quad 1$ & L & & & 0 & 0 & 0 & & & & 0 & 0 & 0 & 0 & 0 & 0 & \\
\hline & 0 & 0 & 0 & 0 & 1 & 0 & 0 & 0 & 0 & 0 & 00 & 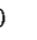 & & & 1 & 1 & 0 & & & & 0 & 0 & 0 & 0 & 0 & 0 & \\
\hline & 1 & 0 & 0 & 0 & 1 & 0 & 0 & 0 & 0 & 0 & 00 & 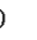 & & & 0 & 0 & 0 & & & & 0 & 0 & 0 & 0 & 0 & 0 & \\
\hline & 1 & 0 & 2 & 0 & 1 & 0 & 1 & 0 & 0 & 0 & 00 & 9 & & & 0 & 0 & 0 & & & & 0 & 0 & 0 & 0 & 0 & 0 & \\
\hline & 0 & 0 & 0 & 2 & 1 & 0 & 1 & 0 & 1 & 0 & 00 & 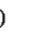 & & & 0 & 0 & 1 & & & & 0 & 0 & 0 & 0 & 0 & 0 & \\
\hline & 0 & 0 & 0 & 1 & 1 & 0 & 0 & 0 & 0 & 0 & 00 & ) & & & 1 & 0 & 0 & & & & 0 & 0 & 0 & 0 & 0 & 0 & \\
\hline & 0 & 0 & 0 & 0 & 1 & 0 & 1 & 1 & 0 & 0 & 00 & 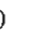 & & & 0 & 1 & 0 & & & & 0 & 0 & 0 & 0 & 0 & 0 & \\
\hline & 0 & 0 & 0 & 0 & 1 & 0 & 0 & 1 & 0 & 0 & 12 & 2 & & & 0 & 0 & 0 & & & & 1 & 0 & 0 & 0 & 0 & 0 & \\
\hline & 2 & 0 & 0 & 0 & 1 & 0 & 0 & 1 & 0 & 0 & 30 & 0 & & & 0 & 0 & 0 & & & & 0 & 0 & 0 & 0 & 0 & 0 & \\
\hline & 3 & 0 & 0 & 2 & 1 & 0 & 1 & 0 & 0 & 0 & 00 & 0 & & & 0 & 0 & 0 & & & & 0 & 0 & 0 & 0 & 0 & 0 & \\
\hline & 0 & 0 & 0 & 0 & 1 & 0 & 0 & 0 & 0 & 0 & 00 & 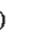 & & & 0 & 0 & 1 & & & & 0 & 0 & 0 & 0 & 0 & 0 & \\
\hline 60 & 0 & 0 & 0 & 0 & 1 & 0 & 0 & 0 & 0 & 0 & 00 & 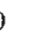 & 15 & 120 & 0 & 0 & 0 & & & & 0 & 0 & 0 & 0 & 0 & 0 & 30 \\
\hline
\end{tabular}


Table 3. Distribution of murres (M), schooling fish (F), and puffins (P) along 3 coastal transects in 1984

\begin{tabular}{|c|c|c|c|c|c|c|c|c|c|c|c|c|c|c|c|c|c|c|c|}
\hline \multirow{2}{*}{$\begin{array}{l}\text { Time } \\
\text { (min) }\end{array}$} & \multicolumn{3}{|c|}{14 Jun } & \multicolumn{3}{|c|}{$8 \mathrm{Jul}$} & \multicolumn{3}{|c|}{$18 \mathrm{Jul}$} & \multirow{2}{*}{$\begin{array}{c}\text { Distance } \\
(\mathrm{km})\end{array}$} & \multirow{2}{*}{$\begin{array}{l}\text { Time } \\
\text { (min) }\end{array}$} & 14 Jun & \multicolumn{3}{|c|}{$8 \mathrm{Jul}$} & & $18 \mathrm{Ju}$ & & Distance \\
\hline & $M$ & $\mathrm{~F}$ & $\mathrm{P}$ & $M$ & F & P & M & F & $\mathrm{P}$ & & & $M \quad F \quad P$ & $M$ & $\mathrm{~F}$ & $\mathrm{P}$ & M & $\mathrm{F}$ & $\mathrm{P}$ & $(\mathrm{km})$ \\
\hline 0 & 0 & 0 & 0 & 0 & 0 & 0 & 0 & 1 & 8 & 0 & & & 50 & 27 & 0 & 0 & 0 & 2 & \\
\hline & 0 & 0 & 0 & 0 & 0 & 0 & 4 & 0 & 2 & & & & 150 & 25 & 0 & 0 & 0 & 0 & \\
\hline & 0 & 0 & 2 & 1 & 0 & 0 & 5 & 2 & 4 & & & & 20 & 16 & 0 & 1 & 0 & 0 & \\
\hline & 0 & 0 & 2 & 1 & 0 & 0 & 37 & 5 & 38 & & & & 2 & 0 & 0 & 0 & 0 & 1 & \\
\hline & 0 & 0 & 0 & 2 & 0 & 0 & 0 & 59 & 48 & & & & 0 & 0 & 0 & 1 & 40 & 8 & \\
\hline & 0 & 0 & 0 & 0 & 0 & 1 & 0 & 0 & 36 & & & & 0 & 0 & 0 & 2 & 5 & 0 & \\
\hline & 0 & 0 & 0 & 0 & 0 & 0 & 0 & 0 & 4 & & & & 0 & 3 & 1 & 0 & 13 & 2 & \\
\hline & 0 & 1 & 0 & 0 & 0 & 26 & 0 & 0 & 37 & & & & 2 & 1 & 0 & 0 & 0 & 1 & \\
\hline & 0 & 4 & 0 & 0 & 0 & 10 & 0 & 0 & 56 & & & & 1 & 0 & 0 & 1 & 0 & 0 & \\
\hline & 0 & 1 & 0 & 0 & 0 & 30 & 0 & 2 & 0 & & & & 0 & 0 & 0 & 4 & 0 & 6 & \\
\hline & 0 & 1 & 0 & 0 & 0 & 28 & 0 & 0 & 0 & & & & 0 & 0 & 0 & & & & \\
\hline & 0 & 1 & 0 & 0 & 0 & 120 & 0 & 0 & 0 & & & & 2 & 0 & 1 & & & & \\
\hline & 0 & 0 & 0 & 0 & 0 & 20 & 0 & 2 & 0 & & & & 0 & 0 & 0 & & & & \\
\hline & 0 & 1 & 0 & 2 & 0 & 0 & 0 & 1 & 0 & & & & 0 & 0 & 0 & & & & \\
\hline & 0 & 1 & 0 & 5 & 0 & 2 & 0 & 0 & 0 & & & & 0 & 0 & 0 & & & & \\
\hline & 0 & 1 & 0 & 141 & 0 & 0 & 0 & 1 & 0 & & & & 0 & 0 & 0 & & & & \\
\hline & 0 & 0 & 0 & 14 & 0 & 0 & 0 & 0 & 1 & & & & 0 & 0 & 0 & & & & \\
\hline & 0 & 1 & 0 & 1 & 0 & 0 & 0 & 0 & 0 & & & & 1 & 4 & 0 & & & & \\
\hline & 0 & 0 & 0 & 8 & 0 & 0 & 0 & 0 & 0 & & & & 1 & 0 & 0 & & & & \\
\hline 20 & 0 & 0 & 0 & 0 & 0 & 3 & 0 & 0 & 0 & 5 & 80 & & 0 & 1 & 0 & & & & 20 \\
\hline & 0 & 1 & 0 & 10 & 0 & 0 & 8 & 0 & 0 & & & & 0 & 0 & 0 & & & & \\
\hline & 0 & 0 & 0 & 12 & 0 & 70 & 11 & 1 & 0 & & & & 0 & 0 & 1 & & & & \\
\hline & 0 & 0 & 0 & 7 & 0 & 0 & 20 & 0 & 0 & & & & 1 & 0 & 0 & & & & \\
\hline & 0 & 0 & 0 & 5 & 0 & 2 & 37 & 0 & 0 & & & & 0 & 1 & 0 & & & & \\
\hline & 0 & 0 & 0 & 8 & 0 & 0 & 18 & 0 & 0 & & & & 0 & 0 & 0 & & & & \\
\hline & 0 & 1 & 0 & 0 & 0 & 0 & 2 & 0 & 4 & & & & 0 & 0 & 0 & & & & \\
\hline & 0 & 4 & 0 & 0 & 0 & 0 & 1 & 0 & 2 & & & & 0 & 0 & 0 & & & & \\
\hline & 0 & 0 & 0 & 0 & 0 & 0 & 0 & 0 & 2 & & & & 0 & 0 & 0 & & & & \\
\hline & 0 & 0 & 0 & 0 & 0 & 0 & 34 & 0 & 0 & & & & 0 & 0 & 0 & & & & \\
\hline & 0 & 1 & 9 & 0 & 0 & 0 & 1 & 0 & 0 & & & & 0 & 0 & 0 & & & & \\
\hline & 0 & 4 & 20 & 0 & 0 & 0 & 0 & 0 & 0 & & & & 0 & 0 & 0 & & & & \\
\hline & 0 & 4 & 15 & 1 & 0 & 0 & 3 & 0 & 0 & & & & 0 & 0 & 0 & & & & \\
\hline & 0 & 1 & 50 & 0 & 0 & 0 & 1 & 0 & 1 & & & & 0 & 0 & 0 & & & & \\
\hline & 4 & 1 & 20 & 1 & 0 & 0 & 2 & 1 & 0 & & & & 0 & 0 & 0 & & & & \\
\hline & 1 & 2 & 3 & 0 & 0 & 0 & 11 & 0 & 1 & & & & 0 & 0 & 0 & & & & \\
\hline & 1 & 1 & 0 & 0 & 0 & 0 & 0 & 1 & 0 & & & & 0 & 1 & 0 & & & & \\
\hline & 10 & 1 & 0 & 0 & 2 & 0 & 0 & 0 & 1 & & & & 0 & 0 & 0 & & & & \\
\hline & 13 & 1 & 0 & 0 & 0 & 0 & 1 & 5 & 0 & & & & 0 & 0 & 0 & & & & \\
\hline & 4 & 0 & 0 & 0 & 0 & 2 & 0 & 1 & 0 & & & & 0 & 0 & 0 & & & & \\
\hline 40 & 2 & 0 & 0 & 0 & 0 & 1 & 1 & 0 & 0 & 10 & 100 & & 0 & 0 & 0 & & & & 25 \\
\hline & 0 & 0 & 0 & 3 & 0 & 1 & 2 & 2 & 0 & & & & 0 & 0 & 0 & & & & \\
\hline & 5 & 0 & 0 & 7 & 1 & 0 & 0 & 0 & 0 & & & & 0 & 0 & 0 & & & & \\
\hline & 5 & 1 & 0 & 7 & 0 & 0 & 0 & 0 & 0 & & & & 0 & 0 & 0 & & & & \\
\hline & 0 & 0 & 0 & 4 & 0 & 0 & 0 & 0 & 0 & & & & 0 & 0 & 0 & & & & \\
\hline & 0 & 1 & 0 & 0 & 1 & 0 & 4 & 0 & 0 & & & & 0 & 0 & 0 & & & & \\
\hline & 0 & 0 & 0 & 2 & 0 & 1 & $\hat{0}$ & 0 & 0 & & & & 0 & 0 & 0 & & & & \\
\hline & 0 & 0 & 0 & 0 & 0 & 0 & 6 & 1 & 0 & & & & 0 & 0 & 1 & & & & \\
\hline & 2 & 1 & 0 & 0 & 0 & 0 & 2 & 0 & 0 & & & & 0 & 0 & 0 & & & & \\
\hline & 0 & 1 & 0 & 0 & 2 & 0 & 0 & 1 & 0 & & & & 0 & 0 & 0 & & & & \\
\hline & 0 & 1 & 0 & 0 & 0 & 0 & 2 & 0 & 0 & & & & 0 & 0 & 3 & & & & \\
\hline & 0 & 0 & 0 & 0 & 0 & 0 & 0 & 9 & 0 & & & & 0 & 1 & 0 & & & & \\
\hline & 1 & 0 & 0 & 0 & 0 & 0 & 0 & 0 & 0 & & & & 1 & 1 & 1 & & & & \\
\hline & 0 & 0 & 0 & 0 & 2 & 0 & 2 & 1 & 0 & & & & 5 & 0 & 2 & & & & \\
\hline & 0 & 0 & 0 & 0 & 51 & 0 & 0 & 2 & 0 & & & & 0 & 0 & 1 & & & & \\
\hline & 0 & 0 & 0 & 87 & 36 & 0 & 0 & 1 & 0 & & & & 0 & 0 & 1 & & & & \\
\hline & 0 & 0 & 0 & 100 & 4 & 0 & 1 & 2 & 0 & & & & 0 & 0 & 0 & & & & \\
\hline & 0 & 1 & 0 & 0 & 4 & 0 & 0 & 0 & 0 & & & & 0 & 0 & 0 & & & & \\
\hline & 0 & 0 & 0 & 8 & 1 & 0 & 0 & 0 & 0 & & & & 0 & 0 & 1 & & & & \\
\hline & 0 & 3 & 0 & 22 & 50 & 1 & 0 & 0 & 0 & & & & 0 & 1 & 2 & & & & \\
\hline 60 & 0 & 1 & 0 & 51 & 50 & 0 & 1 & 0 & 0 & 15 & 120 & & & & & & & & 30 \\
\hline
\end{tabular}




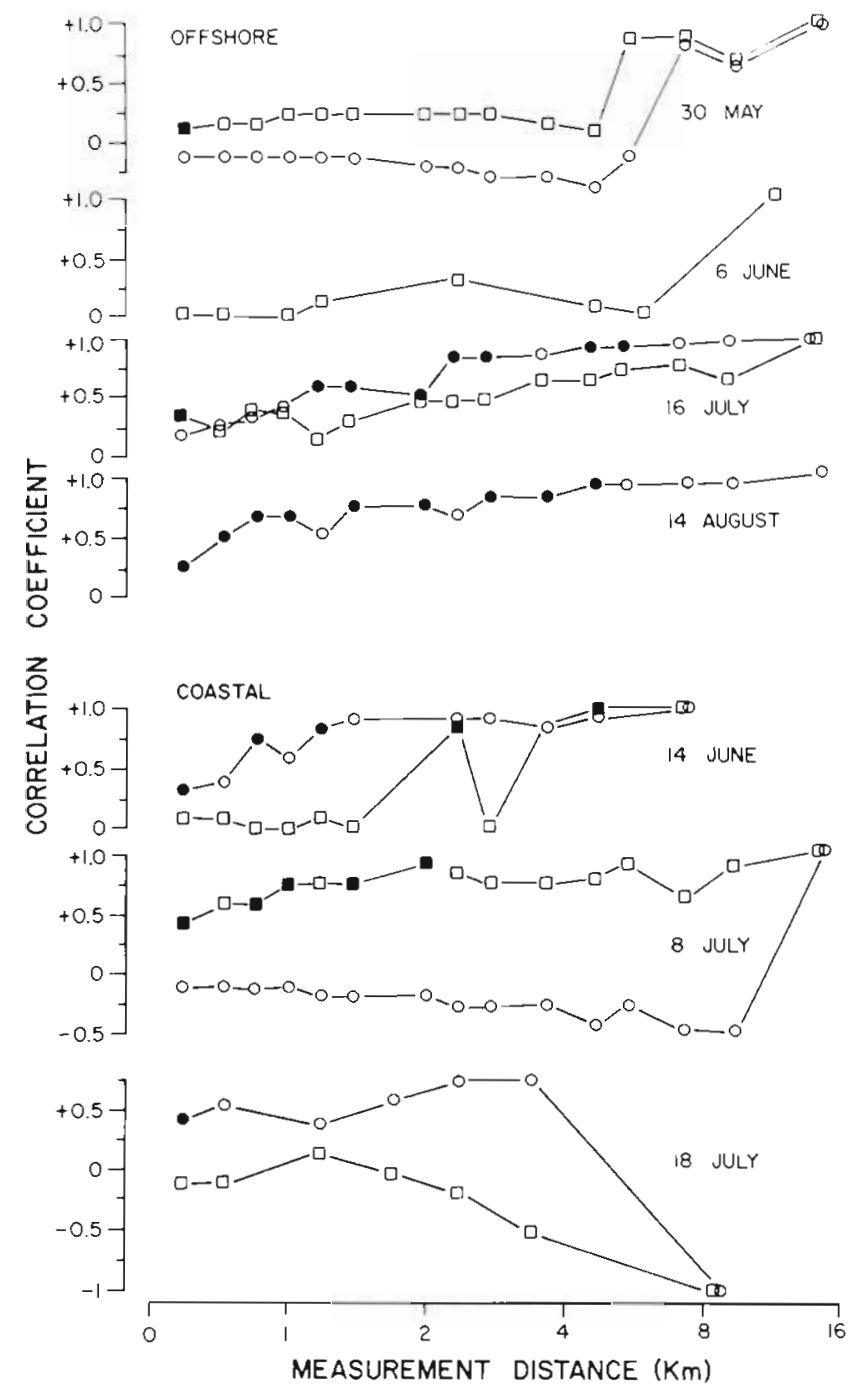

Fig. 3. Correlation of murres (squares) and puffins (circles) with schooling fish, as a function of measurement distance along coastal and offshore transects. Statistical significance as in Fig. 3

second null hypothesis, no correlation at a scale of $0.25 \mathrm{~km}$, averaged $3.5 \%$ (Table 4 ). Type I error for the third null hypothesis, scale independent correlation, was $4.8 \%$ (upper tail) and $5.1 \%$ (lower tail) in murres. For puffins, Type I error was $6.4 \%$ in the upper tail and $4.3 \%$ in the lower tail. Type I error was no greater at large measurement distances than at smaller distances (Table 4), so we concluded that randomization tests were not biased at small sample sizes, which correspond to large measurement distances.

\section{Spatial variation and correlation as a function of measurement distance}

Variability in murres, puffins, and schooling fish during the first survey of the offshore transect was significantly higher at large measurement distances than at the minimum measurement distance, $0.25 \mathrm{~km}$ (Fig. 2). Significant increase in variance-to-mean ratios occurred in fish in 3 of 4 surveys of the offshore transect, and in 2 of 3 surveys of the coastal transect (Table $5)$. Scale-dependent increase in variability occurred in murres during 2 of 3 surveys of the offshore transect, and during 2 of 3 surveys of the coastal transect. Scaledependent increase in variability of puffins occurred during all 6 of the surveys where puffins were present in any number.

Patch scale, based on the maximum significant variance-to-mean ratio, varied from survey to survey. Patch scale ranged from 2.5 to $15 \mathrm{~km}$ in puffins, from 3 to $8.75 \mathrm{~km}$ in murres, and from 1.25 to $15 \mathrm{~km}$ in schooling fish (Table 5). We expected the patch scale of puffins and murres to be similar to the patch scale of fish within surveys. Patch scale of puffins was similar to patch scale of schooling fish (within a factor of 2) during 2 of 3 offshore surveys, and during 2 of 3 coastal surveys (Table 5). Patch scale of murres was similar to patch scale of schooling fish during 2 out of 3 coastal surveys. Patch scale of birds and schooling fish differed by a factor of 2 or more in 3 of the 9 cases where comparison was possible (Table 5).

Significant correlation of murres with schooling fish, at the minimum measurement distance of $0.25 \mathrm{~km}$, was observed during 2 of 3 offshore surveys, and during 1 of 3 coastal surveys (Table 6). Significant correlation of puffins with fish, at the same scale, was observed during 1 of 3 offshore surveys, and during 2 of 3 coastal surveys. The highest correlations at this scale were observed along the coastal transect.

Correlation of murres and puffins with fish was scale-dependent, as indicated by significant increases in correlation with increase in measurement distance along both offshore and coastal transects (Fig. 3). Scale-dependent correlation of murres with fish was observed during 2 of the 3 coastal surveys, but was not observed during offshore surveys. Scale-dependent correlation of puffins with fish was observed during 1 of the 3 coastal surveys, and during 2 of 3 offshore surveys. Larger scale $(>1 \mathrm{~km})$ correlation in the absence of finer scale correlation was observed in puffins during the 16 July survey of the offshore transect. Larger scale correlation accompanied by finer scale correlation of puffins with fish was observed during a subsequent survey of this transect. Finer scale correlation in the absence of larger scale correlation was observed in puffins during the first survey of the longshore transect.

Tracking scale, based on the maximum significant correlation coefficient, ranged from 2.0 to $6 \mathrm{~km}$ (Table 6). Tracking scale and patch scale of birds were similar (within a factor of 2) during the third offshore survey 
Table 4. Type I error for randomization tests of scale-dependent variability, based on 100 randomizations of data from the first offshore surver. 30 May 1984. Values are number of Type I errors (erroneous rejection of null hypothesis in favor of the alternative hypothesis at $\mathrm{p}=0.05$ ) in 100 trials. nt: no test. $\%$ is error per 100 trials

\begin{tabular}{|c|c|c|c|c|c|c|c|c|}
\hline \multirow{2}{*}{$\begin{array}{l}\text { Measureramat } \\
\text { distance (km) }\end{array}$} & & & & \multicolumn{2}{|c|}{$H_{A}: I(0.25)>0$} & \multicolumn{2}{|c|}{$H_{A}: r(0.25)<0$} & \multirow[t]{2}{*}{$\%$} \\
\hline & & & & Murre & Puffin & Murre & Puffin & \\
\hline \multirow[t]{3}{*}{0.25} & & & & 11 & 3 & 0 & 0 & 3.5 \\
\hline & \multicolumn{3}{|c|}{$\mathrm{H}_{\mathrm{A}}: \mathrm{I}^{\prime}(0.25)<\mathrm{I}^{\prime}(\mathrm{F})$} & \multicolumn{2}{|c|}{$\mathrm{H}_{\mathrm{A}}: \mathrm{I}(0.25)<\mathrm{r}(\mathrm{F})$} & \multicolumn{2}{|c|}{$\mathrm{H}_{\mathrm{A}}: \mathrm{r}(0.25)>\mathrm{r}(\mathrm{F})$} & \\
\hline & Murre & Puffin & Fish & Murre & Puffin & Murre & Puffin & \\
\hline 0.50 & 2 & 4 & 1 & 2 & 11 & 3 & 1 & 3.4 \\
\hline 0.75 & 7 & 9 & 1 & 6 & 11 & 3 & 7 & 6.3 \\
\hline 1.0 & 4 & 9 & 3 & 7 & 9 & 4 & 3 & 5.6 \\
\hline 1.25 & 3 & 5 & 2 & 4 & 7 & 8 & 1 & 4.3 \\
\hline 1.5 & 6 & 7 & 6 & 2 & 6 & 6 & 9 & 6.0 \\
\hline 2.0 & 1 & 5 & 3 & 6 & 5 & 4 & 6 & 4.3 \\
\hline 2.5 & 2 & 5 & 4 & 7 & 6 & 8 & 2 & 2.4 \\
\hline 3.0 & 3 & 6 & 3 & 5 & 5 & 6 & 6 & 4.9 \\
\hline 3.75 & 5 & 7 & 4 & 3 & 4 & 6 & 7 & 5.1 \\
\hline 5.0 & 5 & 8 & 2 & 4 & 1 & 8 & 3 & 4.4 \\
\hline 6.0 & 8 & 6 & 8 & 5 & 5 & 8 & 6 & 6.6 \\
\hline 7.5 & 6 & 2 & 5 & 2 & 9 & 5 & 7 & 5.1 \\
\hline 10.0 & 3 & 2 & 3 & 3 & 8 & 2 & 2 & 3.3 \\
\hline 15.0 & 3 & 4 & 3 & nt & nt & nt & $\mathrm{nt}$ & 2.5 \\
\hline Total & 58 & 79 & 48 & 67 & 90 & 71 & 60 & \\
\hline$\%$ & 4.1 & 5.6 & 3.4 & 4.8 & 6.4 & 5.1 & 4.3 & 4.8 \\
\hline
\end{tabular}

and the first coastal survey (Tables 5 \& 6). Tracking scale and patxh scale of birds differed by more than a factor of 2 during the fourth offshore survey and the second coastal survey.

Table 5. Maximum variance-to-mean ratio, $\mathrm{I}^{\prime}(\mathrm{F})$, of murres, puffins, and scholing fish along strip survey. ' indicates I' $(F)$ significantly greater than $I^{\prime}(0.25)$ at $p=0.05$. Patch scale is corresponding measurement distance $(\mathrm{km})$, if $\mathrm{I}^{\prime}(\mathrm{F})$ significant

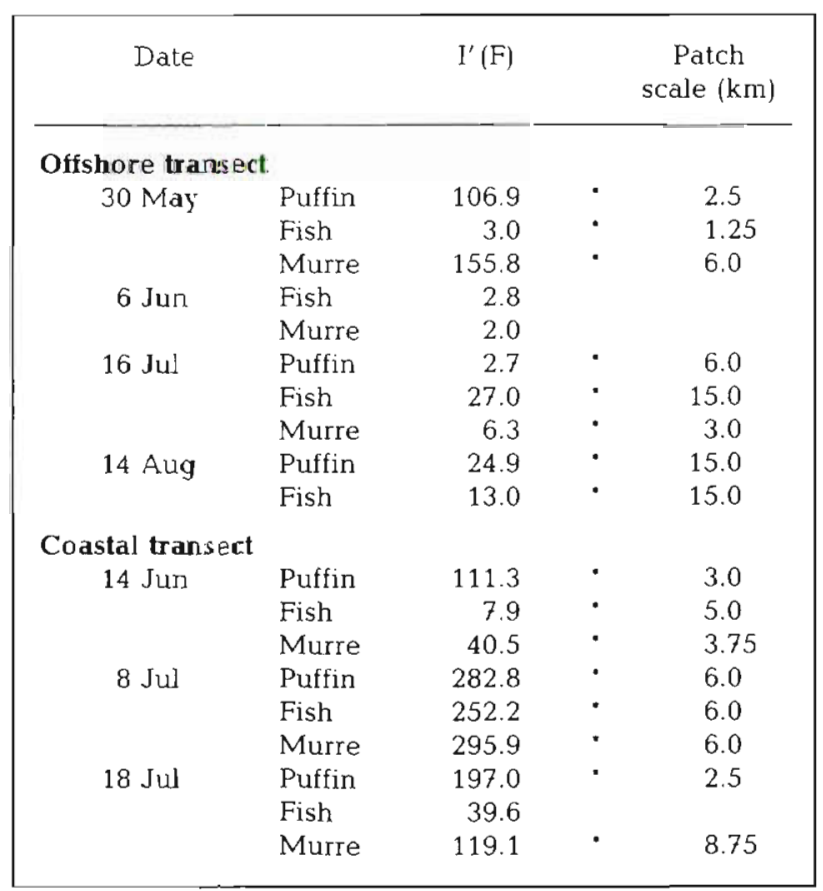

\section{DISCUSSION}

Repeated survey of 2 intersecting transects near the Witless Bay seabird colony showed that extended aggregations of murres and puffins were associated with extended aggregations of schooling fish. Patch scale of birds and fish was similar (within a factor of 2)

Table 6. Correlation of murres and puffins with schooling fish along offshore and coastal transects. $r(0.25)$ is Pearson product-moment correlation coefficient for $0.25 \mathrm{~km}$ (1 $\mathrm{min}$ ) counts. ${ }^{\cdot}$ indicates $H_{A}: r(0.25)>0$ accepted at $p=0.05 . r(F)$ is maximum significant correlation coefficient, $H_{A}: r(0.25)$ $<\mathrm{r}(\mathrm{F})$ accepted at $\mathrm{p}=0.05$. Tracking scale is corresponding measurement distance $(\mathrm{km})$

\begin{tabular}{|c|c|c|c|c|c|}
\hline \multicolumn{2}{|l|}{ Date } & $r(0.25)$ & & $r(F)$ & $\begin{array}{l}\text { Tracking } \\
\text { scale }(\mathrm{km})\end{array}$ \\
\hline \multicolumn{6}{|c|}{ Offshore transect } \\
\hline \multirow[t]{2}{*}{30 May } & Puffin & 0.04 & \multirow{3}{*}{$\cdot$} & \multirow{5}{*}{0.93} & \multirow{5}{*}{6.0} \\
\hline & Murre & 0.10 & & & \\
\hline $6 \mathrm{Jun}$ & Murre & 0.008 & & & \\
\hline \multirow[t]{2}{*}{$16 \mathrm{Jul}$} & Puffin & 0.14 & \multirow[b]{2}{*}{$\cdot$} & & \\
\hline & Murre & 0.30 & & & \\
\hline 14 Aug & Puffin & 0.28 & $\cdot$ & 0.88 & 5.0 \\
\hline \multicolumn{6}{|c|}{ Coastal transect } \\
\hline \multirow[t]{2}{*}{14 Jun } & Puffin & 0.35 & \multirow[t]{2}{*}{$\cdot$} & 0.89 & 2.5 \\
\hline & Murte & 0.07 & & 1.0 & 5.0 \\
\hline \multirow[t]{2}{*}{$8 \mathrm{Jul}$} & Puffin & -0.05 & \multirow{4}{*}{$\dot{.}$} & & \multirow{4}{*}{2.0} \\
\hline & Murre & 0.44 & & 0.90 & \\
\hline \multirow[t]{2}{*}{$18 \mathrm{Jul}$} & Puffin & 0.42 & & & \\
\hline & Murre & -0.05 & & & \\
\hline
\end{tabular}


in 6 out of 9 cases where comparison was possible. Murres and puffins tracked prey at scales on the order of 2 to $6 \mathrm{~km}$. Our transects were too short to detect tracking at scales larger than $6 \mathrm{~km}$.

Extended aggregations of murres and puffins were not simply reflections of extended aggregations of schooling fish. In 3 of 9 comparisons patch scale of birds and schooling fish differed by more than 2 -fold. Also, the observed tracking scale, 2 to $6 \mathrm{~km}$, was generally less than the observed patch scale of predators. This can result from any of several mechanisms, including preferential foraging within extended aggregations of prey, or inability of birds to track rapidly moving prey over distances greater than several $\mathrm{km}$. The interactions of seabirds with their prey needs to be investigated at a scale of tens of kilometres. Foraging interactions at this scale may differ from those reported at smaller scales (Hoffman et al. 1981).

We found that the correlation between birds and schooling fish was stronger over distances of a $\mathrm{km}$ or more than over lesser distances in 5 out of 12 cases. Scale-dependent correlation with prey has been reported for zooplankton (Mackas \& Boyd 1979, Star \& Mullin 1981) and has been hypothesized for marine birds (Hunt \& Schneider in press). Reduced correlation at a scale of hundreds of meters can result from any of several mechanisms, including local avoidance of diving birds by schooling fish, differences in the locomotory capacities of diving birds and schooling fish, and sit-and-wait predation by diving birds within extended aggregations. Capelin were the only abundant schooling fish at Witless Bay during the study, and the reported swimming behaviour of capelin in spawning condition could have reduced finer scale correlation. Offshore feeding schools of capelin descend rapidly out of the surface layer after dawn (Pitt 1958), but capelin in spawning condition occur either as rapidly swimming schools in the surface layer (Atkinson \& Carscadden 1979) or as dense relatively stationary masses near beaches (Sleggs 1933) or within a few metres of subtidal spawning beds (Baake \& Bjorke 1973, Saetre \& Gjosaeter 1975). Alcids, especially murres, may forage on subtidal spawning aggregations, but this appears to be more important during the early morning (Piatt \& Nettleship 1985), than later in the morning, when transects were surveyed. During the day, rapid lateral movements of capelin schools in the nearshore surface layer may have reduced local tracking by alcids.

Three different techniques have been used to investigate spatial correlation and cross-correlation of marine organisms: spectral analysis (Platt \& Denman 1975), auto-correlation analysis (Jumars et al. 1977), and variance-distance curves (Cox \& Isham 1980) scaled to a Poisson process (Angel \& Angel 1967,
Schneider \& Duffy 1985). The procedures used are different, but all 3 produce estimates of covariance functions, whose use has been described by Matern (1960). The equivalence between the first 2 methods (via a Fourier transform) has been noted in the marine literature (Mackas 1984). The equivalence of the first and third methods was noted by Ripley (1978), who showed that constructing a variance-distance curve according to the method of Grieg-Smith (1964) is equivalent to constructing a periodogram using square waves, rather than the trigonometric functions used for standard spectral analyses.

Field data often do not meet the assumptions underlying a given method and this raises questions of reliability (Type I error) and sensitivity (Type II errar). Our analysis of Type I error showed that randomization tests on scaled variance-distance curves were robust to bias at large measurment distances (Fig. 3, Table 4). Randomization tests on scaled covariance-distance curves constructed from bird and fish data were also reliable (Table 4). Randomization tests have been found to be reliable for methods based on auto-correlation analysis (Sokal 1979) and presumably would prove to be reliable for spectral analysis of non-normal data.

The sensitivity (Type II error) of the 3 methods has not been compared. Sokal (1979) found that randomization tests on spatial correlograms were most sensitive when correlation is linear. An informal sensitivity analysis based on simulations (Schneider \& Duffy 1985) showed that variance-distance curves can detect at least 2 types of spatial structure, exponential attenuation with distance from a central point (as in birds), and regular spacing within a patch having a discrete border (as in schooling fish).

Randomization tests require far more computer time than standard parametric or non-parametric analyses, so we investigated Type I error of Spearman rank correlation tests for increase in variability ( $\mathrm{I}^{\prime}[\mathrm{F}]$ ) with increase in scale $(F)$. The Type I error, for the 100 trials in Table 4, was $51 \%$ for murres, $43 \%$ for puffins, and $49 \%$ for schooling fish. Type I error for the third hypothesis, change in bird-fish association with change in scale, was $40 \%$ for puffins and $49 \%$ for murres, using the same 100 trials. This was far higher than the expected error rate, $5 \%$, so we concluded that non-parametric methods were not a reliable way to test for scale-dependence in our data.

The correlation between alcids and schooling fish at Witless Bay was scale-dependent and highly variable from survey to survey along the same transect. This has implications for the design and analysis of transect studies of mobile marine organisms. Correlation was intermittent and this means that repeated surveys may be necessary to detect interaction between mobile pre- 
dators and prey. Correlation was scale-dependent and this means that short transects, or long transects with coarse resolution, may fail to detect significant correlation between seabirds and their prey. Similarly, analysis at a single measurement period (e.g. a $10 \mathrm{~min}$ count) may fail to detect large or smaller scale association. Recent studies on murres (Woodby 1984) and terns (Safina \& Burger 1985) have reported no significant association between seabirds and nekton. Correlation was tested at a single scale in these studies, ca 3 $\mathrm{km}$ in murres and ca $0.5 \mathrm{~km}$ in terns. Association at a different scale may have gone undetected in both studies. A more complete discussion of scale-dependence and its implications for the collection and analysis of transect data can be found in Denman \& Mackas (1978).

The interaction of mobile populations of predators with their prey depends on the form of the aggregative response to prey abundance (Murdoch \& Oaten 1975), but little is known about the aggregative response of marine vertebrates to their prey. We found that the strength of association between seabirds and schooling fish varied with spatial scale. This means that spatial scale needs to be considered in the analysis of the strength and form of aggregative response to prey by highly mobile predators such as birds.

Acknowledgements. This work was funded by the Department of Supply and Services of Canada (Contract \#03ST. FP001-3-2165), the Canadian Wildlife Service, Fisheries and Oceans Canada, and the Newfoundland Institute for Cold Ocean Science. We thank V. Mercer, D. A. Methven, O. Quinn, and J. McKnight for assistance in data collection and reduction. The manuscript was improved by comments from B. Collins, A. J. Gaston, D. A. Methven, W.A. Montevecchi, P. Pepin, and $\mathrm{H}$. Whitehead. This is NICOS contribution No. 196

\section{LITERATURE CITED}

Angel, M. V., Angel, H. H. (1967). Distribution pattern analysis in a marine benthic community. Helgoländer Wiss. Meeresunters. 15: 445-454

Atkinson, D. B., Carscadden, J. E. (1979). Biological characteristics of inshore capelin, Mallotus villosus (Muller), June-July 1977. Fisheries and Marine Service Technical. Report 881: 1-18. Dept. Fisheries and Oceans, St. John's, Newfoundland, Canada.

Baake, S., Bjorke, H. (1973). Diving observations on Barents Sea capelin at the spawning grounds off northern Norway. FiskDir. Skr. Ser. HavUnders. 16: 140-147

Briggs, K. T., Dettman, K. F., Lewis, D. B., Tyler, W. B. (1984). Phalarope feeding in relation to autumnal upwelling off California. In: Nettleship, D. N., Sanger, G. A., Springer, P. F. (ed.) Marine birds: their feeding ecology and commercial fisheries relationships. Can. Wildlife Serv. Spec. Pub., Ottawa, p. 51-64

Brown, R. G. B. (1980). Seabirds as marine animals. In: Burger, J., Olla, B. L., Winn, H. E. (ed.) Behavior of marine animals, Vol. 4. Plenum Press, New York, p. 1-39

Brown, R. G. B., Nettleship, D. N. (1984). Capelin and sea- birds in the northwest Atlantic. In: Nettleship, D. N., Sanger, G. A., Springer, P. F. (ed.) Marine birds: their feeding ecology and commercial fisheries relationships. Canadian Wildlife Service Spec. Pub., Ottawa. p. 184-194

Cox, D. R., Isham, V (1980). Point processes. Chapman and Hall, London

Denman, K. L., Mackas, D. L. (1978). Collection and analysis of underway data and related physical measurements. In: Steele, J. H. (ed.) Spatial pattern in plankton communities. Plenum Press, New York, p. 85-109

Forbes, S. T., Nakken, O. (1972). Manual of methods for fisheries resource survey and appraisal, Part 2. The use of acoustic instruments for fish detection and abundance estimation. FAO Manuals in Fisheries Science 5: 1-138

Grieg-Smith, P. (1964). Quantitative plant ecology, 2nd edn. Butterworths, London

Hoffman, W., Heinemann, D., Wiens, J. A. (1981). The ecology of seabird feeding flocks in Alaska. Auk 98: 437-456

Hunt, G. L., Jr., Schneider, D. (in press). Scale dependent processes in the physical and biological environment of marine birds. In: Croxall, J. P. (ed.) Seabird feeding ecology: the role of seabirds in marine ecosystems. Cambridge University Press, Cambridge

IMSL (1982). International Mathematics and Statistics Library, Houston, Texas, USA

Jumars, P. A., Thistle, D., Jones, M. L. (1977). Detecting twodimensional spatial structure in biological data. Oecologia (Berl.) 28: 109-123

Kinder, T. H., Hunt, G. L., Schneider, D., Schumacher, J. D. (1983). Correlation between seabirds and oceanic fronts around the Pribilof Isiands, Alaska. Estuar. coast. Shelf Sci. 16: 309-319

Mackas, D. L. (1984). Spatial autocorrelation of plankton community composition in a continental shelf ecosystem. Limnol. Oceanogr. 29: 451-471

Mackas, D. L., Boyd, C. M. (1979). Spectral analysis of zooplankton spatial heterogeneity. Science 204: 62-64

Mahoney, S. P. (1979). Breeding biology and behaviour of the Common Murre (Uria aalge aalge [Pont]) on Gull Island, Newfoundland. M. Sc. thesis, Memorial University, St. John's Newfoundland

Matern, B. (1960). Spatial variation. Stochastic models and their application to some problems in forest surveys and other sampling investigations. Medd. fran Statens Skogsforskningsinstitute 49: 1-144

Murdoch, W. W., Oaten, A. (1975). Predation and population stability. Adv. ecol. Res. 9: 2-132

Piatt, J. F., Nettleship, D. N. (1985). Diving depths of four alcids. Auk 102: 293-297

Pitt, T. K. (1958). Distribution, spawning and racial studies of the capelin, Mallotus villosus (Muller), in the offshore Newfoundland area. J. Fish. Res. Bd Can. 15: 275-293

Platt, T., Denman, K. L. (1975). Spectral analysis in ecology. Ann. Rev. Ecol. Syst. 6: 189-210

Reed, W. J. (1983). Confidence estimation of ecological aggregation indices based on counts - a robust procedure. Biometrics 39: 987-998

Ripley, B. D. (1978). Spectral analysis and the analysis of pattern in plant communities. J. Ecol. 66: 965-981

Saetre, R., Gjosaeter, J. (1975). Ecological investigations on the spawning grounds of Barents Sea capelin. FiskDir. Skr. Ser. HavUnders. 16: 203-227

Safina, C., Burger, J. (1985). Common tern foraging: seasonal trends in prey fish densities and competition with bluefish. Ecology 66: 1457-1463

Schneider, D. (1982). Fronts and seabird aggregations in the southeastern Bering Sea. Mar. Ecol. Prog. Ser. 10: 101-103 
Schneider, D., Duffy, D. C. (1985). Scale-dependent variability in seabird abundance. Mar Ecol. Prog. Ser 25: $211-218$

Schreider, Y. A. (1966). The Monte Carlo method. Pergamon Press, Oxford

Sleggs, G. F. (1933). Observations upon the economic biology of the capelin (Mallotus villosus O. F. Muller). Rep. Newf. Fishery Res. Lab 1(2): 1-66

Sokal, R. R. (1979). Testing statistical significance of geographic variation patterns. Syst. Zool. 28: 227-232
Star, J. L., Mullin, M. M. (1981). Zooplankton assemblages in three areas of the North Pacific as revealed by continuous horizontal transects. Deep Sea Res. 28: 1303-1322

Templeman, W. (1948). The life history of the caplin (Mallotus villosus O. F. Muller) in Newfoundland waters. Nfld. Gov. Lab. Res. Ser., Bull. 17: 1-151

Woodby, D. (1984). The April distribution of murres and prey patches in the southeastern Bering Sea. Limnol. Oceanogr. 29: 181-188

This article was submitted to the editor; it was accepted for printing on June 30, 1986 